\title{
Room Temperature Strong Infra-Red Light Emission of Boron Doped Silicon Nanowires
}

\author{
F. Fabbri, ${ }^{*}$ L. Lazzarini,* G. Salviati, ${ }^{*}$, N. Fukata** \\ * IMEM-CNR, Viale G.B. Usberti 37/A, University Campus, 43010 Parma, Italy \\ ** International Center for Materials Nanoarchitectonics, National Institute for Materials Science \& \\ PRESTO JST, 1-1 Namiki, Tsukuba, Ibaraki, 305-0044 (Japan)
}

Silicon nanowires (Si-NWs) are promising for different fields of science such as sensing applications and nano-electronics [1][2]. The possibility of having strong light emission from these nanostructures can open new scenario in optoelectronics, in telecommunication engineering and also in the possible integration with commercial silicon based electronics. The homogeneous doping along the NWs represents a key milestone for a variety of electronic and optoelectronic devices [1] [3]. In this work we present the evidence of deep infra-red emission of boron doped Si-NWs at room temperature studied by cathodoluminescence spectroscopy. A throughout correlation with structural characterization is carried out in order to identify the origin of this strong emission. Boron doped Si-NWs are grown by means of a chemical vapour deposition with silane $\left(\mathrm{SiH}_{4}\right)$ as silicon precursor and diborane $\left(\mathrm{B}_{2} \mathrm{H}_{6}\right)$ as the precursor for boron doping. The analyzed samples are grown with different diborane pressure in order to dope the NWs with different amounts of boron atoms. Cathodoluminescence (CL) spectroscopy of bundle of NWs is employed to analyze the light emission in a large range of wavelengths $(400-1800 \mathrm{~nm})$, with different measurement conditions as beam current and temperature.High resolution transmission electron microscopy (HRTEM), and micro-Raman spectroscopy, are used to assess the structural and chemical properties of the NWs. Figure 1 shows a secondary electron image (SEM) of the dense forest of nanowires analyzed by cathodoluminescence spectroscopy in Fig. 2 where a typical spectrum in the infrared for boron doped Si NWs is reported, a broad emission peaked at $0.8 \mathrm{eV}$ with a shoulder on the high energy side is found. Accurate Gaussian deconvolution evidences four components, set at $0.98 \mathrm{eV}, 0.92$ $\mathrm{eV}, 0.85 \mathrm{eV}$ and $0.78 \mathrm{eV}$ respectively. These emissions are widely known in the bulk silicon luminescence literature, named D-bands. The origin of these $\mathrm{D}$ has been are under debate for over two decades, and proposing different explanations have been proposed such as heavy metals impurities, dislocation kink or oxygen precipitates [4][5]. The correlation between optical and structural properties of the NWs is discussed in terms of the boron precursor pressure.

References

[1] Y. Li, F. Qian, J. Xiang, and C.M. Lieber Material Today 9 (10) (2006) 18

[2] F. Patolsky, B.P. Timko, G. Zheng, and C.M. Lieber, MRS Bulletin 32 (2007) 142

[3] N. Fukata, Advanced Material 21 (27) (2009) 2829

[4] A J Kenyon et al J. Phys.: Condens. Matter 15 (2003) S2843

[5] S Binetti et al J. Phys.: Condens. Matter 14 (2002)13247 


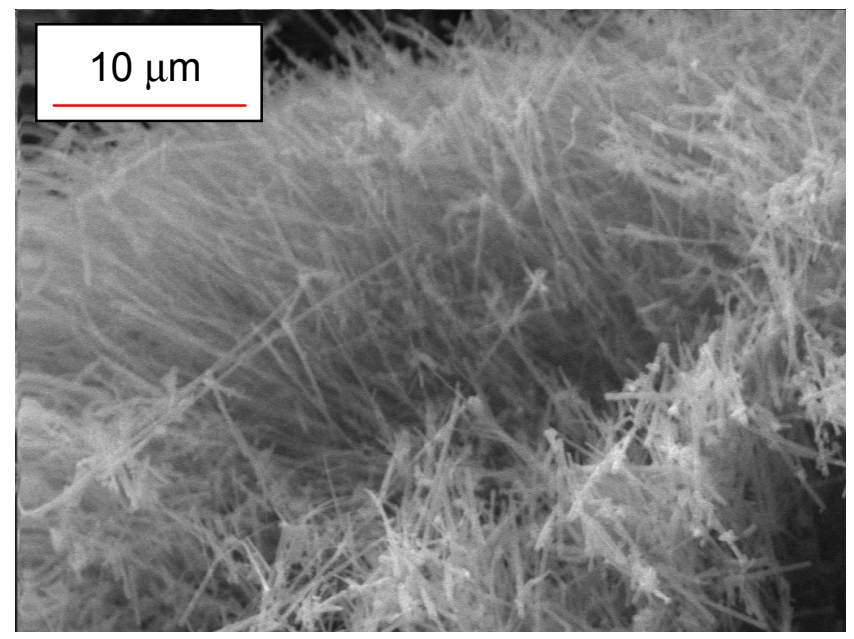

FIG. 1. SEM image of the bundle of Si NWs studied by means of cathodoluminescence spectroscopy

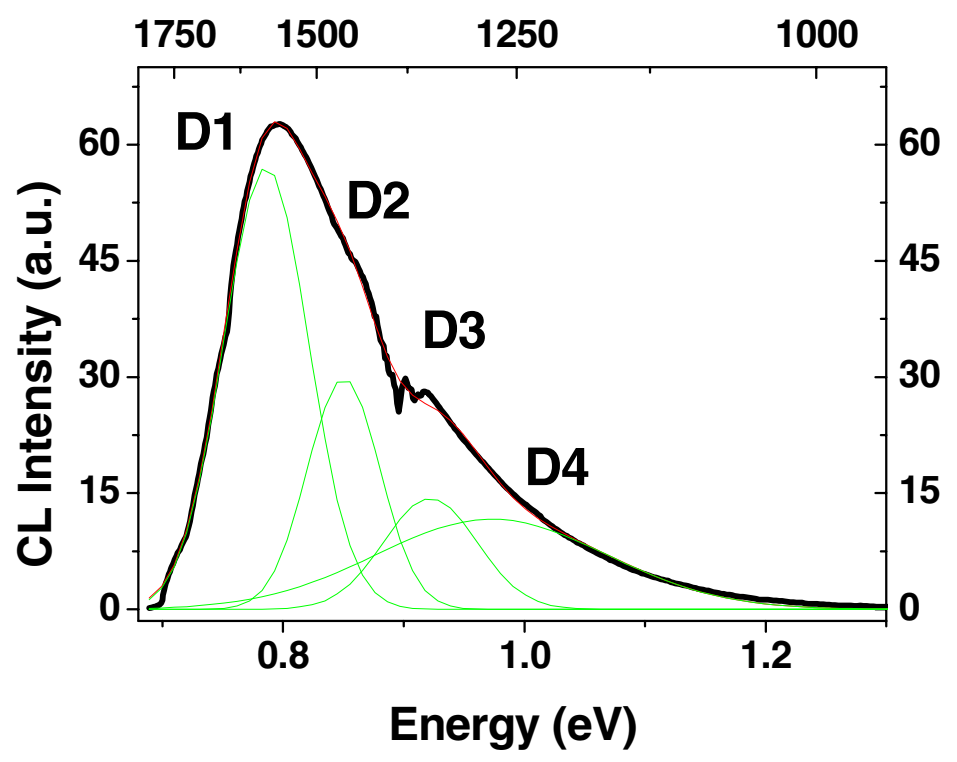

FIG. 2. Typical room temperature CL spectrum of boron doped Si-NWs, reporting a strong broad emission peaked at $0.8 \mathrm{eV}$. Accurate Gaussian deconvolution revelas four components set at 0.98 $\mathrm{eV}, 0.92 \mathrm{eV}, 0.85 \mathrm{eV}$ and $0.78 \mathrm{eV}$. The energy positions are in agreement with the D-bands found in bulk silicon. 